\title{
Notes on density matrix perturbation theory
}

Cite as: J. Chem. Phys. 153, 164105 (2020); https://doi.org/10.1063/5.0022244

Submitted: 20 July 2020 . Accepted: 25 September 2020 . Published Online: 23 October 2020

Lionel A. Truflandier, Rivo M. Dianzinga, and (D) David R. Bowler

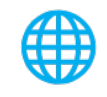

\section{ARTICLES YOU MAY BE INTERESTED IN}

A self-consistent field formulation of excited state mean field theory

The Journal of Chemical Physics 153, 164108 (2020); https://doi.org/10.1063/5.0019557

Electronic structure software

The Journal of Chemical Physics 153, 070401 (2020); https://doi.org/10.1063/5.0023185

Quadratically convergent self-consistent field of projected Hartree-Fock

The Journal of Chemical Physics 153, 164103 (2020); https://doi.org/10.1063/5.0025280

\section{Meet the Next Generation of Quantum Analyzers And Join the Launch Event on November 17th}

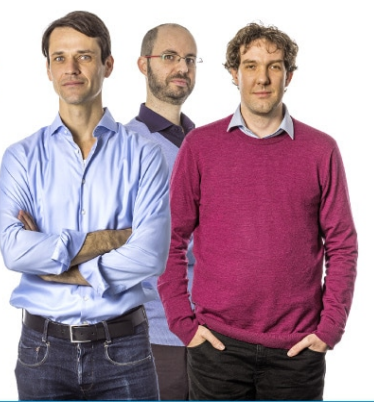




\title{
Notes on density matrix perturbation theory
}

\author{
Cite as: J. Chem. Phys. 153, 164105 (2020); doi: 10.1063/5.0022244 \\ Submitted: 20 July 2020 - Accepted: 25 September 2020 • \\ Published Online: 23 October 2020

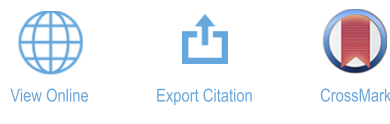

Lionel A. Truflandier, ${ }^{1, a)}$ (D) Rivo M. Dianzinga,' and David R. Bowler ${ }^{2,3}$ (D)

\begin{abstract}
AFFILIATIONS
${ }^{1}$ Institut des Sciences Moléculaires (ISM), Université Bordeaux, CNRS UMR 5255, 351 cours de la Libération, 33405 Talence Cedex, France

${ }^{2}$ London Centre for Nanotechnology, UCL, 17-19 Gordon St., London WC1H OAH, United Kingdom and Department of Physics and Astronomy, UCL, Gower St., London WC1E 6BT, United Kingdom

${ }^{3}$ International Centre for Materials Nanoarchitechtonics (MANA), National Institute for Materials Science (NIMS), 1-1 Namiki, Tsukuba, Ibaraki 305-0044, Japan
\end{abstract}

a) Author to whom correspondence should be addressed: lionel.truflandier@u-bordeaux.fr

\begin{abstract}
Density matrix perturbation theory (DMPT) is known as a promising alternative to the Rayleigh-Schrödinger perturbation theory, in which the sum-over-states (SOS) is replaced by algorithms with perturbed density matrices as the input variables. In this article, we formulate and discuss three types of DMPT, with two of them based only on density matrices: the approach of Kussmann and Ochsenfeld [J. Chem. Phys. 127, 054103 (2007)] is reformulated via the Sylvester equation and the recursive DMPT of Niklasson and Challacombe [Phys. Rev. Lett. 92, 193001 (2004)] is extended to the hole-particle canonical purification (HPCP) from Truflandier et al. [J. Chem. Phys. 144, 091102 (2016)]. A comparison of the computational performances shows that the aforementioned methods outperform the standard SOS. The HPCP-DMPT demonstrates stable convergence profiles but at a higher computational cost when compared to the original recursive polynomial method.
\end{abstract}

Published under license by AIP Publishing. https://doi.org/10.1063/5.0022244

\section{INTRODUCTION}

Traditionally, analytical evaluation of the response of a system to a perturbation is based on the Rayleigh-Schrödinger perturbation theory (RSPT), taking the form of a sum-over-states (SOS) that requires knowledge of the full set of eigenstates. Recent emerging developments have seen the SOS replaced with the response equation, resolved using density matrices as the working variables along with matrix-matrix multiplication-rich recursion algorithms such as kernel polynomials. ${ }^{1}$ Working directly with density matrices is of great interest since we can exploit their natural property of sparsity, which is the key point in designing linear scaling approaches (though we leave the application of sparsity to this work to a future publication). Another advantage is found in the fact that matrixmatrix multiplication can be efficiently parallelized using message passing interface (MPI) or optimized on graphical processing units (GPUs).

The first applications of the RSPT to molecular-orbital (MO) wave function-based self-consistent-field (SCF) methods were introduced during the 1960s for the computation of molecular properties such as magnetic susceptibility, ${ }^{2}$ static polarizabilities, and force constants, ${ }^{3,4}$ which are all related to second-order energy derivatives through the calculation of the first-order change of the wave functions with respect to the perturbation. Similar to the unperturbed case, variational solutions of the perturbed MOs are obtained by solving the so-called coupled-perturbed self-consistent field (CPSCF) equations. ${ }^{5-7}$ These early developments based on either the perturbed molecular-orbitals or the mixed perturbed atomicorbitals/molecular-orbitals (AOs/MOs) were well known to involve cumbersome, especially at that time, matrix transformations. ${ }^{8,9}$ In 1962, McWeeny had already introduced the elegant formalism of the density matrix perturbation theory (DMPT) ${ }^{10,11}$, extended to the CPSCF equations resolution by Diercksen and McWeeny ${ }^{12}$ for the evaluation of $\pi$-electron polarizabilities using the Pariser-ParrPople model. ${ }^{13-15}$ Note that the DMPT formulation of McWeeny still required a SOS and, thus, the knowledge of the eigenstates. This work has first inspired Moccia to generalize the McWeeny-CPSCF equation resolution to the non-orthogonal basis. ${ }^{16,17}$ Perturbationdependent non-orthogonal basis implementation was then proposed by Dodds, McWeeny, Sadlej, and Wolinski ${ }^{18-20}$ for the calculation of atomic (hyper)-polarizabilities using the Hartree-Fock method in conjunction with the Gaussian-type orbital basis sets. The 
advantages of McWeeny's approach over AOs/MOs-CPSCF have been clearly outlined, for instance, in the seminal article of Wolinski, Hinton, and Pulay ${ }^{21}$ dealing with the calculation of magnetic shieldings.

In comparison to the RSPT-SOS, density matrix based methods were introduced more recently, around the year 2000. Ochsenfeld and Head-Gordon first reformulated the CPSCF equations in terms of the density matrix only ${ }^{22}$ (referred to as D-CPSCF by the authors) starting from the Li-Nunes-Vanderbilt (LNV) unconstrained energy functional, ${ }^{23}$ where the McWeeny purification polynomial $^{24,25}$ is used as the input density matrix. Later, Kussmann and Ochsenfeld recognized the important deficiencies in this initial version, which were corrected for in the alternative derivation in Refs. 26 and 27. Within the same spirit, Larsen et al. ${ }^{28}$ followed by Coriani et al. ${ }^{29}$ have derived and implemented, respectively, the response equations using the asymmetric Baker-CampbellHausdorff expansion ${ }^{30}$ for the auxiliary density matrix. Within the field of density matrix purifications, Niklasson, Weber, and Challacombe have introduced a recursive variant of the $\mathrm{DMPT}^{31-33}$ based on the purification spectral projection method detailed in Ref. 34 . Whereas their theoretical framework is general, that is, any recursive polynomial expansion respecting constraints imposed by the density matrix properties may be considered, only performances of the second-order polynomial trace-correcting purification ${ }^{34}$ (TC2)also referred to as second-order spectral projection ${ }^{35,36}$ - have been investigated. 37,38

Recently, we had introduced a Lagrangian formulation for the constrained minimization of the $N$-representable density matrix ${ }^{39}$ based on the McWeeny idempotency error functional. ${ }^{24}$ Within the canonical ensemble (NVT), this gave rise to a unique traceconserving recursive polynomial purification that can be recast in terms of the hole-particle duality condition. The closed-form of this hole-particle canonical purification (HPCP) makes it self-consistent, that is, heuristic adjustment of the polynomial during the course of the purification is not required. Moreover, providing an adequate initial guess, the HPCP is variational and monotonically convergent.

Following the pioneer work of Niklasson and Challacombe, ${ }^{3}$ our current aim is to develop a robust and performant purification based DMPT using the HPCP. In this paper, we are mainly concerned to (i) review the SOS-McWeeny-DMPT, the Kussmann and Ochsenfeld formulation of DMPT (later referred to as Sylvester-DMPT, vide infra), and the purification-DMPT, (ii) derive DMPT equations based on the HPCP density matrix kernel using an orthogonal representation, and (iii) perform a fair comparison of the computational efficiency of the aforementioned methods.

\section{THEORETICAL BACKGROUND}

\section{A. The one-electron density matrix}

We consider an ensemble of fermions at the thermodynamical equilibrium in the external potential created by the nuclei. Given a set of $N$ occupied states, whose wave function is written in the form of a single determinant, the general expression for the spinless oneparticle density operator is

$$
\hat{D}=\sum_{i} \rho_{i}\left|\psi_{i}\right\rangle\left\langle\psi_{i}\right|
$$

where $\left\{\rho_{i}\right\}$ are the occupation numbers associated with the oneelectron states $\left\{\psi_{i} \mid\left\langle\psi_{i} \mid \psi_{j}\right\rangle=\delta_{i j}\right\}$, the latter being, for instance, eigenvectors of any one-electron model Hamiltonian in a tight-binding approach or the Fock operator when dealing with a self-consistent field method as found in the Hartree-Fock or Kohn-Sham meanfield approximation. Hereafter, it shall be denoted by $\hat{H}$, irrespective of the approach. Given $\hat{H}$ for $\hat{D}$ to describe a stationary state within the NVT ensemble, the necessary and sufficient conditions are

$$
\begin{gathered}
{[\hat{H}, \hat{D}]=0, \quad \text { subject to }} \\
\hat{D}=\hat{D}^{\dagger}, \\
0 \leq \rho_{i} \leq 1, \\
\sum_{i} \rho_{i}=N,
\end{gathered}
$$

where $[\cdot, \cdot]$ is the usual symbol for the commutator of two operators. Note that the hermicity constraint in $(2 \mathrm{~b})$ is already enforced by definition (1). However, if we are interested in solving $\hat{D}$ directly, without the support of the eigenstates, this condition will have to be imposed at the beginning and during the iterative resolution. As a result, Eq. (2) mainly expresses that $\hat{H}$ and $\hat{D}$ must share the same eigenstates subject to the $N$-representability constraints, which are: (i) no more than two electron can occupy a given state, assuming spin paired electrons, (ii) the total number of electrons $(2 N)$ is fixed. If now, we want to guarantee that $\hat{D}$ corresponds to the ground state, i.e., the lowest energy states are filled up to the Fermi level $\left(\epsilon_{\mathrm{f}}\right)$ and allows for fractional occupation around $\epsilon_{\mathrm{f}}$, we should combine conditions (2) with the Fermi-Dirac (FD) distribution

$$
\hat{D}=\left(I+e^{\beta(\hat{H}-\mu I)}\right)^{-1},
$$

where $\beta=\left(k_{\mathrm{B}} T\right)^{-1}$ is the inverse electron temperature and the chemical potential, $\mu$, is chosen to conserve the number of electrons. Note that definition (3) is a substitute for Eq. (1) if we want to circumvent the explicit calculation of the eigenstates. The FD distribution also demonstrates that, for non-degenerate systems, there exists a correspondence between the density and the Hamiltonian operator. Unfortunately, its nonlinear character prevents a direct resolution of $\hat{D}$ in terms of $\hat{H}$. This has motivated the introduction of the density matrix polynomial expansion in the 1990s to resolve $\hat{D}$ recursively with $\hat{H}$ as the input. ${ }^{40,41}$ In this work, we shall reduce the theoretical framework to pure states where the occupation numbers of the eigenstates are either 0 or 1 . This leads to

$$
\hat{D}=\sum_{i \in \text { occ }}\left|\psi_{i}\right\rangle\left\langle\psi_{i}\right|,
$$

where, compared to Eq. (1), the subset of occupied states is sufficient to fully determine the one-particle density matrix. In that case, the $N$-representability conditions of Eqs. (2c)-(2d) can be recast as

$$
\begin{gathered}
\hat{D}=\hat{D}^{2}, \\
\operatorname{Tr}\{\hat{D}\}=N,
\end{gathered}
$$


that is, the density matrix of the pure state is idempotent. The corresponding ground-state is determined by the zero temperature limit of Eq. (3),

$$
\hat{D}=\Theta(\mu I-\hat{H}) \quad \text { with } \quad \lim _{T \rightarrow 0} \mu=\epsilon_{\mathrm{f}},
$$

with $\Theta$ the Heaviside step function. If we now consider a separable Hilbert space of dimension $M(\geq N)$ that admits an orthonormal basis $\left\{\phi_{\mu} \in L^{2} \mid\left\langle\phi_{\mu} \mid \phi_{v}\right\rangle=\delta_{\mu \nu}\right\}_{\mu=1}^{M}$, the one-particle density operator of Eq. (4) has the following matrix representation:

$$
D=\sum_{i \in \mathrm{occ}} P_{i} \quad \text { with } \quad P_{i}=c_{i} \otimes c_{i},
$$

where $\left\{c_{i} \in \mathbb{R}^{M \times 1} \mid c_{i}^{\dagger} c_{j}=\delta_{i j}\right\}_{i=1}^{N}$ are the column vectors containing the expansion coefficients such that $\left\{\left|\psi_{i}\right\rangle=\sum_{\mu}^{M} c_{\mu i}\left|\phi_{\mu}\right\rangle \mid c_{\mu i}\right.$ $\left.=\left\langle\phi_{\mu} \mid \psi_{i}\right\rangle\right\}_{i=1}^{N}$, and $\left\{P_{i} \in \mathbb{R}^{M \times M} \mid P_{i} P_{j}=P_{i} \delta_{i j}, \operatorname{Tr}\left\{P_{i}\right\}=1\right\}_{i=1}^{N}$ is the set of $N$ orthonormal projectors belonging to the subset of the occupied states. At this stage, we shall introduce the one-hole density matrix built from the subspace of the $\bar{N}$ virtual (unoccupied) states,

$$
\bar{D}=\sum_{i \in \text { virt }} \bar{P}_{i} \quad \text { with } \quad \bar{P}_{i}=\bar{c}_{i} \otimes \bar{c}_{i},
$$

such that $M=\bar{N}+N$. Throughout the paper, quantities related to those states will be indicated by a bar accent. Stationary oneparticle and one-hole density matrices must obey the two following identities:

$$
\begin{gathered}
D+\bar{D}=I, \\
D \bar{D}=0,
\end{gathered}
$$

with $I$ the identity matrix. As a result, it can be easily demonstrated that the one-hole density matrix for a pure state obeys the same properties as its one-particle equivalent, e.g., the idempotency and trace conservation of Eq. (5).

\section{B. One-electron density matrix perturbation theory}

Let us now consider the perturbed one-particle density and the Hamiltonian matrix $D_{\lambda}$ and $H_{\lambda}$, respectively, where $\lambda$ stands for any time-independent perturbation. At the zero electronic temperature limit, for $D_{\lambda}$ to describe the perturbed stationary state corresponding to the unperturbed ground state $D$, it must also obey the following rules:

$$
\begin{gathered}
{\left[H_{\lambda}, D_{\lambda}\right]=0, \quad \text { subject to }} \\
D_{\lambda}=D_{\lambda}^{\dagger}, \\
D_{\lambda}=D_{\lambda}^{2}, \\
\operatorname{Tr}\left\{D_{\lambda}\right\}=N,
\end{gathered}
$$

where Eqs. (11c) and (11d) stand for the $N$-representability conditions. Note that (11b) will be enforced by construction (vide infra). We shall expand the perturbed density and the Hamiltonian matrix in a power series with respect to a perturbation parameter $(0<\lambda \leq 1)$,

$$
\begin{aligned}
& D_{\lambda}=D^{(0)}+\lambda D^{(1)}+\lambda^{2} D^{(2)}+\cdots+\lambda^{k} D^{(k)}, \\
& H_{\lambda}=H^{(0)}+\lambda H^{(1)}+\lambda^{2} H^{(2)}+\cdots+\lambda^{k} H^{(k)},
\end{aligned}
$$

where $X^{(k)}$ represents the $k$ th-order change of the quantity $X$ with respect to $\lambda ; D^{(0)} \equiv D$ and $H^{(0)} \equiv H$ are the unperturbed density and the Hamiltonian matrix, respectively. Inserting expansion (12a) into the $N$-representability constraints of Eqs. (11c) and (11d), and by equating the perturbation orders, yields

$$
\begin{gathered}
D^{2}=D \\
\text { subject to } \quad \operatorname{Tr}\{D\}=N, \\
D D^{(1)}+D^{(1)} D=D^{(1)} \\
\text { subject to } \operatorname{Tr}\left\{D^{(1)}\right\}=0, \\
D D^{(2)}+\left(D^{(1)}\right)^{2}+D^{(2)} D=D^{(2)}, \\
\text { subject to } \quad \operatorname{Tr}\left\{D^{(2)}\right\}=0, \\
\text { subject to } \quad \operatorname{Tr}\left\{D^{(3)}\right\}=0, \\
\quad D^{(1)} D^{(2)}+D^{(2)} D^{(1)}+D^{(3)} D=D^{(3)} \\
\sum_{l=0}^{k} D^{(l)} D^{(k-l)}=D^{(k)} \\
\text { subject to } \quad \operatorname{Tr}\left\{D^{(k)}\right\}=0 .
\end{gathered}
$$

Further repeating the perturbation identification by introducing Eqs. (12a) and (12b) in the commutator of Eq. (11a), we obtain

$$
\begin{gathered}
{[H, D]=0,} \\
{\left[H, D^{(1)}\right]+\left[H^{(1)}, D\right]=0,} \\
{\left[H, D^{(2)}\right]+\left[H^{(1)}, D^{(1)}\right]+\left[H^{(2)}, D\right]=0,} \\
{\left[H, D^{(3)}\right]+\left[H^{(1)}, D^{(2)}\right]+\left[H^{(2)}, D^{(1)}\right]+\left[H^{(3)}, D\right]=0,} \\
\vdots \\
\sum_{l=0}^{k}\left[H^{(l)}, D^{(k-l)}\right]=0 .
\end{gathered}
$$

The generalized idempotency constraint of Eq. (13e) and the stationary condition of Eq. (14e) constitute the working equations for developing the various forms of the density matrix perturbation theory (DMPT), which are described in Secs. II C-II E.

The derivation of the expressions for the observable quantities induced by perturbations as energy contributions in the power series expansion $E_{\lambda}=E^{(0)}+\lambda E^{(1)}+\lambda^{2} E^{(2)}+\cdots+\lambda^{k} E^{(k)}$ can be found in many text books and references. ${ }^{42,43}$ In the case of the tight-binding method, the unperturbed one-electron energy $\left(E^{(0)} \equiv E\right)$ is simply

$$
E=2 \operatorname{Tr}\{H D\} .
$$


By stopping at the first-order in the Hamiltonian expansion of Eq. (12b), it can be shown that the corresponding energy corrections for $k>0$ are given by

$$
E^{(k+1)}=\frac{2}{k} \operatorname{Tr}\left\{H^{(1)} D^{(k)}\right\} .
$$

Consequently, energy derivatives up to the order $(k+1)$ involves knowledge of the density matrices up to the order $k$. A more popular approach, ${ }^{27,44-48}$ for computing energy derivatives for $k>2$, relies on the $(2 k+1)$ Wigner rule, which states that $E^{(2 k+1)}$ can be obtained from the $k$ th-order perturbed wave functions. ${ }^{42}$ It is noteworthy that McWeeny et al., ${ }^{10,12,18,19}$ later reported by Niklasson et al., ${ }^{31-33}$ have adapted the $(2 k+1)$ th theorem to perturbed density matrices as inputs, up to order 4 with respect to the energy, without any support of the wave functions.

\section{SOS-McWeeny-DMPT}

The DMPT equations proposed by McWeeny involve the partitioning of $D^{(k)}$ into four distinct contributions and their resolutions. ${ }^{10}$ Using the closure relation of Eq. (9), any matrix representation of the operator $\hat{O}$ can be expressed into the following projected components:

$$
\begin{aligned}
O=O_{o o}+O_{o v} & +O_{v o}+O_{v v} \\
\text { with } & \\
O_{o o} & =D O D \\
O_{v v} & =\bar{D} O \bar{D} \\
O_{o v} & =D O \bar{D} \\
O_{v o} & =\bar{D} O D .
\end{aligned}
$$

The subscripts $o o$ and $v v$ designate the occupied-occupied and virtual-virtual diagonal contributions, whereas ov and vo stand for the non-diagonal occupied-virtual and virtual-occupied transition terms. To the first order of perturbation, on applying the projection decomposition of Eq. (17) to both sides of Eq. (13b), ${ }^{10}$ we obtain

$$
2 D_{o o}^{(1)}+D_{o v}^{(1)}+D_{v o}^{(1)}=D_{o o}^{(1)}+D_{o v}^{(1)}+D_{v o}^{(1)}+D_{v v}^{(1)},
$$

where, by comparing terms of the left-hand and right-hand sides (abbreviated as lhs and rhs, respectively, in the rest of the text), it can be easily deduced that

$$
D_{o o}^{(1)}=0, \quad D_{v v}^{(1)}=0 .
$$

As a result, the first-order perturbed density matrix is fully determined by the occupied-virtual transition matrix, such that

$$
D^{(1)}=D_{o v}^{(1)}+D_{v o}^{(1)}=D_{o v}^{(1)}+\left(D_{o v}^{(1)}\right)^{\dagger}
$$

Resolving $H^{(1)}$ into four components using Eq. (17), we can search for $D_{o v}^{(1)}$ through Eq. (14b). After simplification, the following working equations are found:

$$
H_{o v}^{(1)}=\left[H, D_{o v}^{(1)}\right] \text { and } H_{v o}^{(1)}=\left[D_{v o}^{(1)}, H\right] .
$$

On recalling the Hermitian property of the unperturbed and perturbed Hamiltonian matrices, the lhs of Eq. (21) is found to be the conjugate transpose of the rhs; then solving one of the two equations is sufficient to evaluate the perturbed density matrix of Eq. (20).

The common Rayleigh-Schrödinger sum-over-states (SOS) is recovered from Eq. (21), by applying the spectral resolution for the non-perturbed Hamiltonian matrix according to

$$
H=\sum_{i \in \mathrm{occ}} \epsilon_{i} P_{i}+\sum_{j \in \text { virt }} \bar{\epsilon}_{j} \bar{P}_{j}
$$

where indices $i$ and $j$ run over the energy-weighted projectors for the occupied and unoccupied space, respectively. On substitution of Eq. (22) into Eq. (21), using the following identity:

$$
O_{o v}=\sum_{i \in \text { occ }} \sum_{j \in \text { virt }} P_{i} O \bar{P}_{j},
$$

we obtain

$$
\begin{gathered}
\sum_{i \in \mathrm{occ}} \sum_{j \in \mathrm{virt}}\left(D_{o v, i j}^{(1)}\left(\epsilon_{i}-\bar{\epsilon}_{j}\right)-H_{o v, i j}^{(1)}\right)=0, \\
\text { with: } \quad D_{o v, i j}^{(1)}:=\left(P_{i} D^{(1)} \bar{P}_{j}\right) \in \mathbb{R}^{M \times M}, \\
H_{o v, i j}^{(1)}:=\left(P_{i} H^{(1)} \bar{P}_{j}\right) \in \mathbb{R}^{M \times M} .
\end{gathered}
$$

This equation can be recast into the following SOS form:

$$
D_{o v}^{(1)}=\sum_{i \in \text { occ }} \sum_{j \in \text { virt }} \frac{H_{o v, i j}^{(1)}}{\epsilon_{i}-\bar{\epsilon}_{j}} .
$$

Using definitions of the one-electron and one-hole projector, Eqs. (7) and (8) respectively, the usual expression of the first-order linear-response of the density matrix is recovered. ${ }^{12,16,18-21}$ In the operator form, it gives

$$
\begin{aligned}
\hat{D}^{(1)}= & \sum_{i \in \mathrm{occ}} \sum_{j \in \text { virt }} \frac{\left\langle\psi_{i}\left|\hat{H}^{(1)}\right| \bar{\psi}_{j}\right\rangle}{\epsilon_{i}-\bar{\epsilon}_{j}}\left|\psi_{i}\right\rangle\left\langle\bar{\psi}_{j}\right| \\
& + \text { conjugate transpose. }
\end{aligned}
$$

Assuming that the sets of non-perturbed eigenvectors, $\left\{\psi_{i}\right\}_{i=1}^{N}$ and $\left\{\bar{\psi}_{j}\right\}_{j=1}^{\bar{N}}$, are properly orthonormalized, then the fact that $\operatorname{Tr}\left\{\left|\psi_{i}\right\rangle\left\langle\bar{\psi}_{j}\right|\right\}=\left\langle\bar{\psi}_{j} \mid \psi_{i}\right\rangle=0 \forall(i, j)$ guarantees the $N$-representability conditions of Eqs. (13a) and (13b).

Derivation for the second- and third-order linear responses is given in Appendix A. From here, we shall briefly review the generalized working equations needed for solving the density matrix response at any order $k>1$. The off-diagonal contributions are given by

$$
\begin{aligned}
D_{o v}^{(k)}= & \sum_{i \in \text { occ }} \sum_{j \in \text { virt }} \frac{H_{o v, i j}^{(k)}-\sum_{l=1}^{k-1}\left[H^{(l)}, D^{(k-l)}\right]_{o v, i j}}{\epsilon_{i}-\bar{\epsilon}_{j}}, \\
& =\sum_{l=1}^{k} \sum_{i \in \text { occ }} \sum_{j \in \text { virt }} \frac{\left[D^{(k-l)}, H^{(l)}\right]_{o v, i j}}{\epsilon_{i}-\bar{\epsilon}_{j}} .
\end{aligned}
$$

In the operator form, it gives

$$
\hat{D}_{o v}^{(k)}=\sum_{l=1}^{k} \sum_{i \in \text { occ }} \sum_{j \in \text { virt }} \frac{\left\langle\psi_{i}\left|\left[\hat{D}^{(k-l)}, \hat{H}^{(l)}\right]\right| \bar{\psi}_{j}\right\rangle}{\epsilon_{i}-\bar{\epsilon}_{j}}\left|\psi_{i}\right\rangle\left\langle\bar{\psi}_{j}\right| .
$$


The diagonal terms are given by

$$
\begin{aligned}
& D_{o o}^{(k)}=-D\left(\sum_{l=1}^{k-1} D^{(l)} D^{(k-l)}\right) D, \\
& D_{v v}^{(k)}=+\bar{D}\left(\sum_{l=1}^{k-1} D^{(l)} D^{(k-l)}\right) \bar{D} .
\end{aligned}
$$

Again here, given a set of orthonormalized non-perturbed eigenvectors, it is easy to show that $\operatorname{Tr}\left\{D_{o v}^{(k)}\right\}=0=\operatorname{Tr}\left\{D_{o o}^{(k)}+D_{v v}^{(k)}\right\}$, ensuring the respect of the generalized perturbed $N$-representability conditions of Eq. (13e). Note that for a Hamiltonian perturbation expansion up to the first order in Eq. (12b), only the commutator $\left[D^{(k-1)}, H^{(1)}\right]$ survives in the $\sum_{l}$ of Eq. (28).

Although McWeeny's formulation of DMPT is based on the density matrix, it still requires the knowledge of the unperturbed eigenstates, which means that at least one Hamiltonian diagonalization must be performed prior entering the DMPT resolution. As it shall be shown below, there exist alternative solutions that allow us to circumvent the expensive diagonalization step.

\section{Sylvester-DMPT}

As in the unperturbed case, to completely bypass the calculation of the eigenstates when solving the DMPT equations, an objective functional with perturbed density matrices as the degree of freedom has to be defined and minimized without the support of the spectral decomposition (22). In this respect, Ochsenfeld and Head-Gordon have proposed to extend the LNV functional ${ }^{23}$ minimization principle to DMPT. ${ }^{22}$ Later, Kussmann and Ochsenfeld reformulated the working equations to cure for numerical instabilities. ${ }^{26,49}$ The approach relies on solving Eq. (14e) subject to commuting with the unperturbed density matrix. For instance, at the first order of perturbation, on multiplying Eq. (14b) from the left and from the right by $D$ separately, and substracting, we obtain ${ }^{50}$

$$
\left[H,\left[D, D^{(1)}\right]\right]+\left[D^{(1)},[D, H]\right]+\left[D,\left[H^{(1)}, D\right]\right]=0 .
$$

Since we assume that the exact zero-order density matrix is known, the second term in Eq. (32) vanishes. By noting that

$$
\left[D,\left[H^{(1)}, D\right]\right]=2 D H^{(1)} D-\left\{D^{2}, H^{(1)}\right\},
$$

with $\{\cdot, \cdot\}$ the symbol for the anticommutator of two operators, then Eq. (32) simplifies to

$$
\left[H,\left[D, D^{(1)}\right]\right]=\left\{D, H^{(1)}\right\}-2 D H^{(1)} D .
$$

A practical form for solving Eq. (34) is obtained by expanding the commutators of the lhs. Using the identity of Eq. (13b) yields

$$
(2 H D-H) D^{(1)}+D^{(1)}(2 D H-H)=\left\{D, H^{(1)}\right\}-2 D H^{(1)} D,
$$

which can be identified as being a Sylvester-like equation of the kind $A X+X B=C,{ }^{51,52}$ where $B:=A^{t}$, and $X:=D^{(1)}$ is the unknown to solve for. ${ }^{53}$ It is worthwhile to note that on multiplying Eq. (34) from the left by $D$, from the right by $\bar{D}$, and conversely by $\bar{D}$ on the left and $D$ on the right, Eq. (21) is recovered. By induction, the DMPT
Eq. (35) can be generalized to any order $k>1$, with the $k$ th-order transition matrix $D^{(k)}$ solution of

$$
\begin{aligned}
{\left[H,\left[D, D^{(k)}\right]\right]=} & \left\{D, H^{(k)}\right\}-2 D H^{(k)} D \\
& +\sum_{l=1}^{k-1}\left[D,\left[D^{(k-l)}, H^{(l)}\right]\right] .
\end{aligned}
$$

As for the first order, by expanding the commutators and using the identity of Eq. (13e), we found

$$
\begin{aligned}
(2 H D & -H) D^{(k)}+D^{(k)}(2 D H-H) \\
& =\left\{D, H^{(k)}\right\}-2 D H^{(k)} D+\sum_{l=1}^{k-1}\left[D,\left[D^{(k-l)}, H^{(l)}\right]\right] \\
& -\left\{D^{(l)} D^{(k-l)}, H\right\} .
\end{aligned}
$$

Assuming that all the lower order (up to $k-1$ ) perturbed density matrices are known on the rhs of the equation, then $D^{(k)}$ on the lhs can be found by solving the Sylvester equation

$$
A X+X A^{t}=C
$$

where $(A, X, C) \in \mathbb{R}^{M \times M}$ are the square matrices. The fixed matrix $A$ corresponds to $(2 H D-H)$ and the fixed matrix $C$ is given by the rhs of Eq. (37). In this work, the algorithm of Bartels and Stewart ${ }^{54}$ (BS) shall be used to solve Eq. (38). Alternatives to the BS algorithm are envisageable by vectorizing Eq. (38), which transforms the Sylvester equation to a standard linear system of equations. Among the numerous iterative methods developed for solving the linear system of equations, ${ }^{55}$ the conjugate-gradient (CG) minimization is one the most efficient, ${ }^{56}$ especially for large scale problems presenting a sparsity pattern. After a set of trials on model systems (cf. Sec. III), we found that the BS algorithm was more efficient than CG minimization.

Note that the CG method is also a popular alternative to iterative diagonalizations, e.g., the Davidson method, ${ }^{57}$ when it is sufficient to access the partial set of the $\tilde{M}$ lowest energy eigenstates, with $N \leq \tilde{M}<M$, as, for instance, for the non-vanishing band gap system, where only the subset of occupied states are required to build $D$ in Eq. (4). This fact is exploited by iterative diagonalizations when the number of basis functions exceeds by far $N$, the typical case being the plane wave (PW) basis set. For insulators, the approximate first $N$ lowest eigenstates in some Krylov subspace of $H$ suffices to achieve the desired accuracy. ${ }^{58,59}$ In this context, bandby-band (also called state-by-state) CG algorithms (BB-CG), ${ }^{59-62}$ which basically perform sequential CG minimizations under some orthonormalization constraints, have also proved to be a valuable alternative. $^{63}$

The BB-CG method is one of the ingredients of the PW-based (density functional) perturbation theory. ${ }^{64-67}$ In contrast to the McWeeny-DMPT, which requires the knowledge of the full eigenspectrum of $H$, it is possible to compute any of the $k$ th-order density matrix, using the sole information available from the occupied eigenstates. This constitutes the framework of the high-order DMPT introduced by Lazzeri and Mauri. ${ }^{68,69}$ The strong overlap existing between this approach, the McWeeny- and the Sylvester-DMPT is discussed in Appendix B. 


\section{E. Purification-DMPT}

A powerful alternative to the McWeeny- and Sylvester-DMPT resides in the density matrix purification method, ${ }^{70}$ which, at the zero order, given the unperturbed Hamiltonian matrix, consists of finding the corresponding ground state density matrix by approaching the Heaviside step function of Eq. (6) using a polynomial recursion. Within the canonical ensemble, it can be formally expressed as

$$
\begin{gathered}
D_{0}=\mathscr{L}_{P}(H ;\{N, \ldots\}), \\
D_{n+1}=\mathscr{F}_{P}\left(D_{n} ;\{N, \ldots\}\right), \\
\text { such that } D_{\infty}=\lim _{n \rightarrow \infty} D_{n},
\end{gathered}
$$

where $D_{0}, D_{n+1}$, and $D_{\infty}$ designate the initial, the $(n+1)$-iterate, and the converged density matrix, respectively. The polynomial recursive sequence is initiated by a linear mapping (39a), where the function $\mathscr{L}_{P}$ rescales, shifts, and reverses the eigenspectrum of $H$ into the proper interval for occupation numbers, i.e., $\forall i: \rho_{i} \in[0,1]$. In that case, the initial guess, $D_{0}$, represents some ground state in the sense of Eq. (1). Then, by applying recursively the polynomial function, $\mathscr{F}_{P}$, the degenerate sets of $N$ and $\bar{N}$ eigenvalues of $D_{n}$ associated with the occupied and virtual subspaces are progressively brought toward 1 and 0 , respectively. These subsets will be symbolized by $\{o\} \equiv\left\{\rho_{i} \mid \lim _{n \rightarrow \infty} \rho_{i}=1\right\}_{i=1}^{N}$ and $\{v\} \equiv\left\{\rho_{i} \mid \lim _{n \rightarrow \infty} \rho_{i}=0\right\}_{i=N+1}^{M}$. At convergence, $D=D_{\infty}$ such that $D$ fulfills the $N$-representability conditions (5) and the ground-state occupation (6) at $T=0$, without prior knowledge of the chemical potential.

In this work, we have considered two different purification schemes, the second-order trace-correcting (TC2) purification, later rebaptized the second-order spectral projection, ${ }^{35}$ and the trace-conserving hole-particle canonical purification (HPCP $)^{39}$. The original TC2 recursive polynomial ${ }^{32,34}$ is given by

$$
\begin{gathered}
\mathscr{F}_{\mathrm{TC} 2}\left(D_{n} ;\{N\}\right)=D_{n}+2\left(\Theta\left(\Delta N_{n}\right)-\frac{1}{2}\right) D_{n} \bar{D}_{n}, \\
\text { with } \Delta N_{n}=N-\operatorname{Tr}\left\{D_{n}\right\} \\
\text { such that } \lim _{n \rightarrow \infty} \Delta N_{n}=0 \\
\text { and } \Theta(x)=\left\{\begin{array}{lll}
0 & \text { if } & x \leq 0 \\
1 & \text { if } & x>0,
\end{array}\right.
\end{gathered}
$$

along with the following initialization mapping:

$$
\mathscr{L}_{\mathrm{TC} 2}(H)=\frac{\tilde{\epsilon}_{\max } I-H}{\tilde{\epsilon}_{\max }-\tilde{\epsilon}_{\min }} .
$$

In the above equation, $\left(\tilde{\epsilon}_{\min }, \tilde{\epsilon}_{\max }\right)$ are the approximated values of the lower and upper bounds of the Hamiltonian matrix eigenspectrum $\left(\epsilon_{\min }, \epsilon_{\max }\right)$, respectively. They can be easily estimated, i.e., without the support of iterative diagonalization, from the Geršgorin's disk theorem ${ }^{71}$ with the following convenient properties: $\tilde{\epsilon}_{\min }<\epsilon_{\min }$ and $\tilde{\epsilon}_{\max }>\epsilon_{\max }$. It should be emphasized that the initial guess generated from Eq. (41) is not $N$-representable since $\operatorname{Tr}\left\{D_{0}\right\}$ is not constrained to be equal to $N$, indeed it must not be. As evidenced by Eq. (40c), the TC2 purification is to be regarded as a discontinuous self-mapping of $D_{n}$, where the $\operatorname{sign}^{72}$ of the deviation of $\operatorname{Tr}\left\{D_{n}\right\}$ with respect to $N$ dictates the polynomial to apply at iteration $n+1$. If at the current state, $\operatorname{Tr}\left\{D_{n}\right\}<N$, all the occupation numbers, but those already trapped at the fixed points 0 and 1 , will be moved (at different rates; the closer to $1 / 2$ the faster) toward the turning point $x_{p}$ $=1$. Conversely, for $\operatorname{Tr}\left\{D_{n}\right\}>N$, the occupation numbers are moved toward $x_{\bar{p}}=0$. In principle, the recursive sequence should be terminated when $\left|\Delta N_{n}\right|$ or/and another relevant convergence criteria is/are below some threshold value, ${ }^{70}$ e.g., $\left\|D_{n} \bar{D}_{n}\right\|$. As a matter of fact, the Heaviside singularity, especially for vanishing-gap systems, may pose some difficulties in achieving proper convergence, since in definition (40c) there is no fixed midpoint for $\Theta(x=0)$. It is likely the cause of numerical instabilities when approaching convergence. In this respect, to cure the possible issues of the TC2 purification, several refinements of Eq. (40b), and the expression of the stopping criteria, have been proposed. ${ }^{35,36,70}$ These refinements, which are more substantial when sparse linear algebra is applied, ${ }^{32,33,38}$ can lead to a significant increase of the algorithmic complexity.

Instead of applying a global upward or downward shift to all the non-converged $\left\{\rho_{i}\right\}$, one can seek to simultaneously operate on $\{o\}$ and $\{v\}$ during the polynomial recursion. The easiest way is to increase the polynomial degree from 2 to 3 , in order to introduce an inflexion point, $\left.x_{\text {flex }} \in\right] 0,1[$, separating the two subsets. For instance, assuming a symmetric model, when half of the available states are occupied, i.e., the filling factor $\theta=N / M$ equals $1 / 2$, and the chemical potential is at the midpoint of $\left[\epsilon_{\min }, \epsilon_{\max }\right], x_{\mathrm{flex}}=1 / 2$ is the optimal position. In this case, all the $\rho_{i} \in\{o\}$ verifying $\rho_{i}>x_{\text {flex }}$ are pushed toward 1, whereas at the same time, all the $\rho_{i} \in\{v\}$ verifying $\rho_{i}<x_{\text {flex }}$ are pushed toward 0 . By recognizing the constant $1 / 2$ in Eq. (40a) as $x_{\text {flex }}$, and on substituting $\Theta$ by $D$ in the same equation, it is intuitively easy enough to see that the resulting polynomial of degree 3 fulfills the requirements stated above. When compared to the TC2 polynomial, both $x_{p}$ and $x_{\bar{p}}$ are now stable fixed points, and more importantly, the iterative mapping is continuous. Actually, it does correspond to the well-known McWeeny recursive sequence, ${ }^{24}$

$$
\begin{aligned}
\mathscr{F}_{\mathrm{McW}}\left(D_{n}\right) & =D_{n}+2\left(D_{n}-\frac{1}{2} I\right) D_{n} \bar{D}_{n} \\
& =3 D_{n}^{2}-2 D_{n}^{3} .
\end{aligned}
$$

Unfortunately, in the general case, unconstrained application of Eq. (42) is likely to deliver $D_{\infty}$ verifying Eq. (2c) but with $\operatorname{Tr}\left\{D_{\infty}\right\}$ $\neq N$, especially when $\theta$ is far from $1 / 2$. Given any $(H, N, M)$, if we search for generalizations of the McWeeny purification, two difficulties must be addressed: (i) how to dynamically adapt $x_{\text {flex }}$ to non-symmetric $\left\{\rho_{i}\right\}$ distributions while maintaining the two stable (un)fixed points (nearby) at 0 and 1; (ii) how to ensure that the converged density matrix is $N$-representable? Although they are not all mandatory, we can consider three constraints under which the problem shall be solved: (a) no a priori information on the structure of $H$ eigenspectrum nor on some of the interior eigenvalues is required, (b) the highest polynomial degree is 3 , and (c) the recursive mapping must remain continuous. A first solution was brought by Palser and Manolopoulos (PM) in their NVT version of the McWeeny purification. ${ }^{75}$ Recently, by solving a constrained optimization problem dealing with the idempotency error minimization of $D,{ }^{39}$ we found that the PM polynomial could be significantly simplified and accelerated through the hole-particle duality. ${ }^{76}$ The resulting hole-particle canonical purification (HPCP) polynomial ${ }^{39}$ is given by 


$$
\begin{gathered}
\mathscr{F}_{\mathrm{HPCP}}\left(D_{n}\right)=D_{n}+2\left(D_{n}-c_{n} I\right) D_{n} \bar{D}_{n}, \\
\text { with } c_{n}=\frac{\operatorname{Tr}\left\{D_{n}^{2} \bar{D}_{n}\right\}}{\operatorname{Tr}\left\{D_{n} \bar{D}_{n}\right\}} \\
\text { such that } \quad \lim _{n \rightarrow \infty} c_{n}=\frac{1}{2},
\end{gathered}
$$

with the linear mapping function

$$
\begin{aligned}
& \mathscr{L}_{\mathrm{HPCP}}(H ;\{N, M, \alpha\})=\left(\alpha \beta_{\min }+(1-\alpha) \beta_{\max }\right) \\
& \times(\tilde{\mu} \mathscr{F}-H)+\theta \mathscr{F}, \\
& \text { with } \quad \tilde{\mu}=\frac{\operatorname{Tr}\{H\}}{M}, \\
& \beta_{\min }=\min \left(\frac{\theta}{\tilde{\epsilon}_{\max }-\tilde{\mu}}, \frac{1-\theta}{\tilde{\mu}-\tilde{\epsilon}_{\min }}\right), \\
& \beta_{\max }=\max \left(\frac{\theta}{\tilde{\epsilon}_{\max }-\tilde{\mu}}, \frac{1-\theta}{\tilde{\mu}-\tilde{\epsilon}_{\min }}\right),
\end{aligned}
$$

where $\alpha$ is the mixing parameter that can be optimized with respect to $\theta .^{39}$ For the numerical experiments presented in Sec. III, $\alpha$ shall be fixed to $1 / 2$. As in the original McWeeny purification, the HPCP polynomial presents an unstable fixed point, $c_{n}$, which in the present case, modulates the position of $x_{\text {flex }}$ and enforces the correction term on the rhs of Eq. (43) to be traceless. Provided a $N$-representable $D_{0}$ using Eq. (44), the HPCP is capable of converging self-consistently to the ground-state density matrix while verifying the $N$-representability conditions throughout the purification process. Note that when approaching convergence, it can be shown $^{75}$ that $c_{n} \rightarrow 1 / 2$. Nevertheless, in this regime, the numerator and denominator of Eq. (43c) tend to zero. As a result, to avoid numerical instabilities related to floating-point round-off, it is safer to fix $c_{n}$ to $1 / 2$ at the very late stage of the purification.

It must be stressed that the TC2 and HPCP methods are very distinct in their NVT minimization principle. For the HPCP, $N$ is kept fixed while $T$ is implicitly minimized, to zero for non-vanishing band-gap system, whereas for the TC2 purification, $T$ is implicitly fixed to zero, and $N$ is perturbatively optimized around the target value, resulting in very different convergence profiles regarding, for instance, monotonicity and variational properties (vide infra).

It is rather remarkable that the perturbed density matrices involved in the power series of $D_{\lambda}$ can also be determined by a straight application of the polynomial recursive sequence (39) with $H_{\lambda}$ as the input. ${ }^{31}$ The perturbed analog of Eq. (39) is formally written as

$$
\begin{array}{r}
\text { initialisation: }\left(\begin{array}{c}
D_{0}^{(0)} \\
D_{0}^{(1)} \\
\vdots \\
D_{0}^{(k)}
\end{array}\right)=\left(\begin{array}{c}
\mathscr{L}_{P}^{(0)}\left(H^{(0)}\right) \\
\mathscr{L}_{P}^{(1)}\left(H^{(1)}\right) \\
\vdots \\
\mathscr{L}_{P}^{(k)}\left(H^{(k)}\right)
\end{array}\right), \\
\text { recursion: }\left(\begin{array}{c}
D_{n+1}^{(0)} \\
D_{n+1}^{(1)} \\
\vdots \\
D_{n+1}^{(k)}
\end{array}\right)=\left(\begin{array}{c}
\mathscr{F}_{P}^{(0)}\left(D_{n}^{(0)}\right) \\
\mathscr{F}_{P}^{(1)}\left(D_{n}^{(0)}, D_{n}^{(1)}\right) \\
\vdots \\
\mathscr{F}_{P}^{(k)}\left(D_{n}^{(0)}, D_{n}^{(1)}, \ldots D_{n}^{(k)}\right)
\end{array}\right),
\end{array}
$$

such that: :

$$
D_{\infty}, D_{\infty}^{(1)}, \ldots, D_{\infty}^{(k)}:=\lim _{n \rightarrow \infty}\left(D_{n}, D_{n}^{(1)}, \ldots, D_{n}^{(k)}\right),
$$

where $\mathscr{L}_{P}^{(0)}$ and $\mathscr{F}_{P}^{(0)}$ correspond to the unperturbed linear mapping and recursive polynomial as introduced in Eqs. (39a) and (39b), respectively. Equation (45) outlines that, provided some set of 0 -to$k$ order Hamiltonian matrices to initialize the 0 -to- $k$ order density matrices, repeated application of the $k$-perturbed recursive sequence delivers better estimates of the $(k+1)$ input quantities by propagating the perturbed purification. Note that the evaluation of the higher order perturbed term does not involve prior exact knowledge of the lower orders, not even the exact unperturbed density matrix; instead, all the orders are resolved on-the-fly. Compared to the standard approaches, e.g., the McWeeny- and Sylvester-DMPT that are based on solving order-by-order $(k+1)$-sets of linear equations with $D^{(k)}$ as unknown, the purification-DMPT performs the resolution of one unique nonlinear equation with $(k+1)$ unknowns. In order to avoid heavy notations, the $(k+1)$-dimension of the inputs and outputs in Eq. (45) will be simplified by retaining only the $k$-order term in the function argument and value, respectively.

By substituting Eq. (12b) into Eq. (41) and Eq. (12a) into Eq. (40a), equating the perturbation orders, the $k$-perturbed component of the TC2 recursive sequence ${ }^{37}$ is written as

$$
\begin{gathered}
\mathscr{L}_{\mathrm{TC} 2}^{(k>0)}\left(H^{(k)}\right)=-\frac{H^{(k)}}{\tilde{\epsilon}_{\max }-\tilde{\epsilon}_{\min }}, \\
\mathscr{F}_{\mathrm{TC} 2}^{(k)}\left(D_{n}^{(k)} ;\{N\}\right)=D_{n}^{(k)}+2\left(\Theta\left(\Delta N_{n}\right)-\frac{1}{2}\right) \sum_{l=0}^{k} D_{n}^{(l)} \bar{D}_{n}^{(k-l)} .
\end{gathered}
$$

By referring to Eq. (13), it is easily seen that the sum over the perturbed hole-particle density matrix product appearing on the rhs of Eq. (46b) corresponds to the error in the idempotency (noted $\Delta D^{(k)}$ below). On remembering that $\bar{D}^{(0)}=I-D^{(0)}$ and $\bar{D}^{(k>0)}=-D^{(k>0)}$, such that, at iterate $n$,

$$
\begin{gathered}
\Delta D_{n}^{(0)}=D_{n}-D_{n}^{2}, \\
\Delta D_{n}^{(1)}=D_{n}^{(1)}-\left(D_{n} D_{n}^{(1)}+D_{n}^{(1)} D_{n}\right), \\
\Delta D_{n}^{(2)}=D_{n}^{(2)}-\left(D_{n} D_{n}^{(2)}+\left(D_{n}^{(1)}\right)^{2}+D_{n}^{(2)} D_{n}\right), \\
\vdots \\
\Delta D_{n}^{(k)}=D_{n}^{(k)}-\sum_{l=0}^{k} D_{n}^{(l)} D_{n}^{(k-l)},
\end{gathered}
$$

we obtain the more compact TC2 perturbed recursion formula

$$
D_{n+1}^{(k) \stackrel{\mathrm{TC} 2}{=}} D_{n}^{(k)}+2\left(\Theta\left(\Delta N_{n}\right)-\frac{1}{2}\right) \Delta D_{n}^{(k)} .
$$

By proceeding the same way with the hole-particle canonical purification initialization and recursive polynomial [Eqs. (44) and (43), respectively], the $k$-perturbed component of the HPCP recursive sequence is written as

$$
\mathscr{L}_{\mathrm{HPCP}}^{(k>0)}\left(H^{(k)} ;\{N\}\right)=-\left(\alpha \beta_{\min }+(1-\alpha) \beta_{\max }\right) H^{(k)},
$$




$$
\begin{aligned}
\mathscr{F}_{\mathrm{HPCP}}^{(k)}\left(D_{n}^{(k)}\right)= & D_{n}^{(k)}+2 \sum_{l=0}^{k} \\
& \times\left(\sum_{m=0}^{l} D_{n}^{(m)} D_{n}^{(l-m)}-c_{n} D_{n}^{(l)}\right) \bar{D}_{n}^{(k-l)} .
\end{aligned}
$$

Using definition (47), after some rearrangement, we find

$$
D_{n+1}^{(k)} \stackrel{\mathrm{HPCP}}{=} D_{n}^{(k)}+2\left(D_{n}-c_{n} I\right) \Delta D_{n}^{(k)}+2 \sum_{l=0}^{k-1} D_{n}^{(k-l)} \Delta D_{n}^{(l)} .
$$

It should be stressed that since the unstable fixed-point in Eq. (50) depends on the unperturbed density matrix, resolving $D^{(k)}$ orderby-order is not possible with the current formalism. A full decoupling of the perturbed purification would require to establish and solve a constrained optimization problem as in Ref. 39, with the $k$ order perturbed idempotency relations (13) as the main ingredient for the quadratic functional form to minimize. This possibility shall be addressed in a further study.

Comparing Eq. (50) to Eq. (48), it is clear that the perturbed HPCP approach involves additional correction terms in the polynomial expansion, increasing its computational complexity with respect to the perturbed TC2. Looking at the most computationally demanding task, ${ }^{77}$ i.e., the number of matrix multiplications (MMs), which in both cases increases linearly with the perturbation order, it is found that the perturbed HPCP requires nearly three times more MMs than the perturbed TC2. ${ }^{78}$ This is balanced out by the rate of convergence, which, as in the unperturbed case, is linear for the TC2, but quadratic for the HPCP.

\section{EXAMPLES AND PERFORMANCES}

To illustrate the perturbation-based DMPT, we have considered two examples taken from the $\pi$-bonding perturbation in Hückel theory. ${ }^{79}$ Our aim here is to test the purification approaches, in terms of both stability and convergence. The first one consists of decomposing the Hückel matrix of the benzene molecule into (i) a non-perturbed Hamiltonian containing the matrix elements of the butadiene and ethylene subunits and (ii) a first-order perturbed Hamiltonian associated with their coupling. The decomposition is explicitly given as

$$
\begin{aligned}
H_{\lambda} \text { (benzene) } & =H^{(0)}+\lambda H^{(1)} \\
& =\left(\begin{array}{llllll}
\alpha & \beta & 0 & 0 & 0 & 0 \\
\beta & \alpha & \beta & 0 & 0 & 0 \\
0 & \beta & \alpha & \beta & 0 & 0 \\
0 & 0 & \beta & \alpha & 0 & 0 \\
0 & 0 & 0 & 0 & \alpha & \beta \\
0 & 0 & 0 & 0 & \beta & \alpha
\end{array}\right)+\lambda\left(\begin{array}{llllll}
0 & 0 & 0 & 0 & 0 & \beta \\
0 & 0 & 0 & 0 & 0 & 0 \\
0 & 0 & 0 & 0 & 0 & 0 \\
0 & 0 & 0 & 0 & \beta & 0 \\
0 & 0 & 0 & \beta & 0 & 0 \\
\beta & 0 & 0 & 0 & 0 & 0
\end{array}\right),
\end{aligned}
$$

where $\alpha$ and $\beta$ are the usual symbols for the carbon Coulomb and resonance integrals, respectively. We note that in this example the perturbation matrix is purely non-diagonal. For the second example, we have chosen the Hückel matrix of the pyridine molecule for which one of the $s p^{2}-\mathrm{C}$ in benzene is substituted by a $s p^{2}-\mathrm{N}$ atom. Considering the benzene Hückel matrix as the zero-order Hamiltonian, the perturbation matrix contains the variation of the Coulomb $(\Delta \alpha)$ and resonance $(\Delta \beta)$ integrals related to the nitrogen/carbon substitution. The corresponding total Hamiltonian is written as

$$
\begin{aligned}
H_{\lambda}(\text { pyridine })= & H^{(0)}+\lambda H^{(1)} \\
= & \left(\begin{array}{ccccccc}
\alpha & \beta & 0 & 0 & 0 & \beta \\
\beta & \alpha & \beta & 0 & 0 & 0 \\
0 & \beta & \alpha & \beta & 0 & 0 \\
0 & 0 & \beta & \alpha & \beta & 0 \\
0 & 0 & 0 & \beta & \alpha & \beta \\
\beta & 0 & 0 & 0 & \beta & \alpha
\end{array}\right) \\
& +\lambda\left(\begin{array}{cccccc}
\Delta \alpha & \Delta \beta & 0 & 0 & 0 & \Delta \beta \\
\Delta \beta & 0 & 0 & 0 & 0 & 0 \\
0 & 0 & 0 & 0 & 0 & 0 \\
0 & 0 & 0 & 0 & 0 & 0 \\
0 & 0 & 0 & 0 & 0 & 0 \\
\Delta \beta & 0 & 0 & 0 & 0 & 0
\end{array}\right),
\end{aligned}
$$

with, for nitrogen, the following parameters: $\Delta \alpha=\beta / 2$ and $\Delta \beta$ $=-0.2 \beta$. For numerical experiments, we set $\alpha$ and $\beta$ to -11.400 and $-2.568 \mathrm{eV}$, respectively. Stopping criteria for purification was based on the norm of the unperturbed density matrix iterates such that the purification process was stopped for $\left\|D_{n+1}-D_{n}\right\|_{\mathcal{F}}<10^{-12}$. Further tests were performed on the Frobenius norms and traces of all the perturbed density matrices to ensure full convergence at all the orders.

In both examples, the perturbation series is expected to converge such that, by setting $\lambda=1$, the sum over the perturbed densities in Eq. (12a) converges toward the exact density matrix $D_{\lambda}$ as obtained from the full Hamiltonian $H_{\lambda}$; obtained, for instance, by diagonalizing $H_{\lambda}$ and summing over the $N$ occupied states as in Eq. (4). The same way, the sum over the perturbed energies, $E_{\text {pert }}$ $=\sum_{k} E^{(k)}$, computed from Eq. (16) must converge toward the exact reference value, $E_{\text {exact }}=2 \operatorname{Tr}\left\{H_{\lambda} D_{\lambda}\right\}$. This is demonstrated for benzene and pyridine in Figs. 1(a) and 2(a), respectively, where $E_{\text {exact }}-$ $E_{\text {pert }}$ are plotted as a function of the number of perturbation terms entering in $\sum_{k} E^{(k)}$, up to $k=20$. For benzene, we note that for symmetry reason, only even orders contribute to $E_{\text {pert }}$. At $k=20$, for benzene, we found that $E_{\text {pert }}$ is converged to within $5 \mathrm{meV}$ for $E_{\text {exact }}$ $=-88.9440 \mathrm{eV}$, compared to a much faster convergence for pyridine with the same level of convergence reach at $k=3$, with $E_{\text {exact }}$ $=-85.1005 \mathrm{eV}$. On the same figures ( $y$-right axis) are also reported the norms of the density matrices $\left\|D_{\infty}^{(k)}\right\|$ as a function of $k$, which confirm the faster convergence of the $\pi$-bonding perturbation in pyridine.

We emphasize that identical results were obtained with the reference McWeeny- and Sylvester-DMPT. Considering the benzene perturbation series at higher order, reliability of the purificationbased DMPT starts to degrade for $k>55$ using HPCP compared to $k>17$ for TC2. This trend has been confirmed for other systems and $\pi$-bonding perturbations (not shown here), indicating that the HPCP purification is more stable than TC2 when increasing the perturbation order. This could be related to the ill-definition of the stopping criteria of TC2, becoming a very sensitive parameter in the extreme conditions of very high perturbation order. Nevertheless, this has a weak interest in practical applications since they generally do not require a perturbed density matrix higher than order 3 .

Evolution of the density matrices (up to order 3) during the purification process is plotted in Figs. 1 and 2, panels (b) for the 


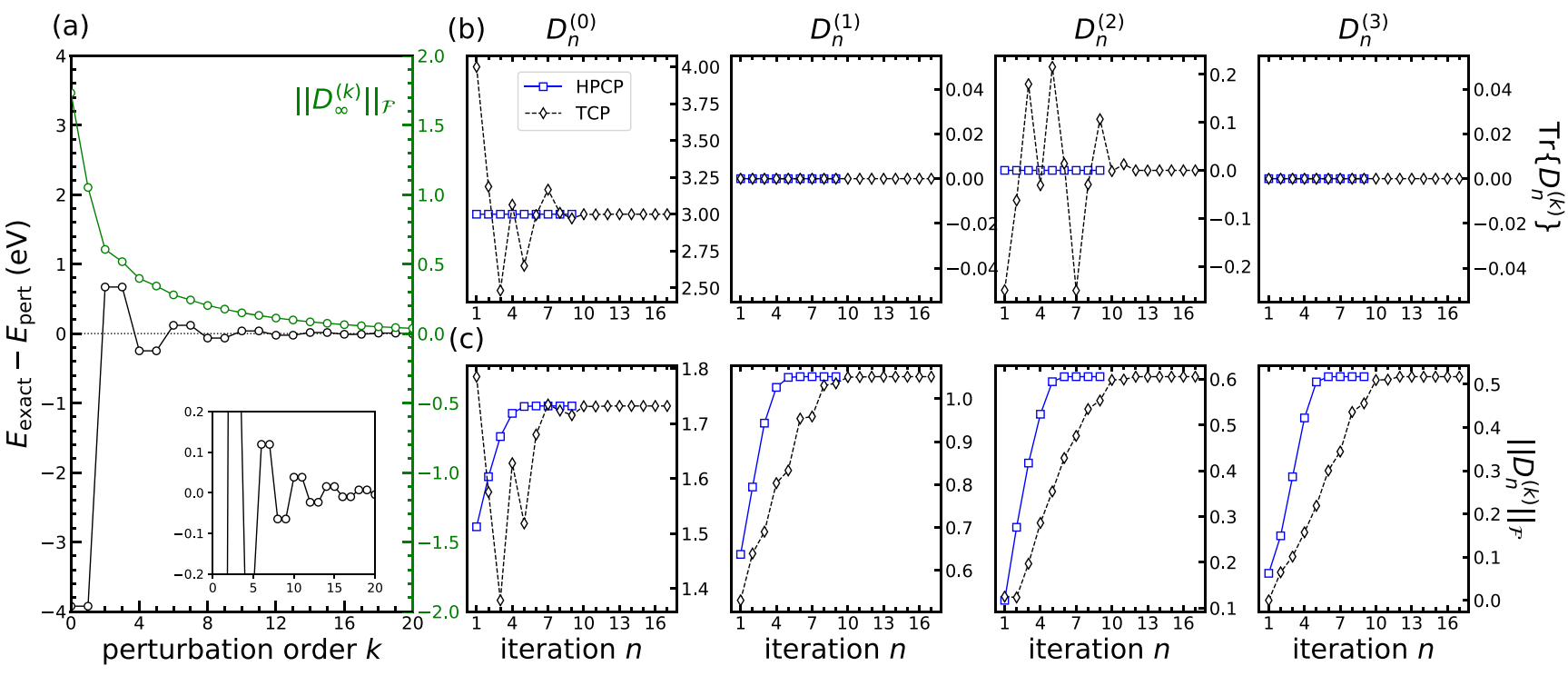

FIG. 1. Perturbation series for $\pi$-bonding in benzene using perturbed Hückel matrices (see text for details). (a) Convergence of the perturbed energy and Frobenius norm of the corresponding perturbed density matrices using purification-DMPT. The convergence of the unperturbed and the first three perturbed density matrices during the purification cycles are presented in terms of their traces (b) and their norms (c), for both the TC2 and HPCP polynomials.

trace and (c) for the norm. First, in both cases, the convergence of the HPCP-DMPT is achieved in fewer iterations compared to TC2 as expected from the convergence profile of the two polynomials (quadratic vs linear, respectively). The property of traceconservation of the zero-order density matrix fulfilled by the HPCP is apparent from these figures. In this respect, the TC2 polynomial demonstrates an erratic behavior with strong oscillations at the beginning of the purification. These oscillations remain present at higher order, especially when looking to the pyridine case. We note that the zero-trace conservation of $D^{(1)}$ and $D^{(3)}$ observed with both

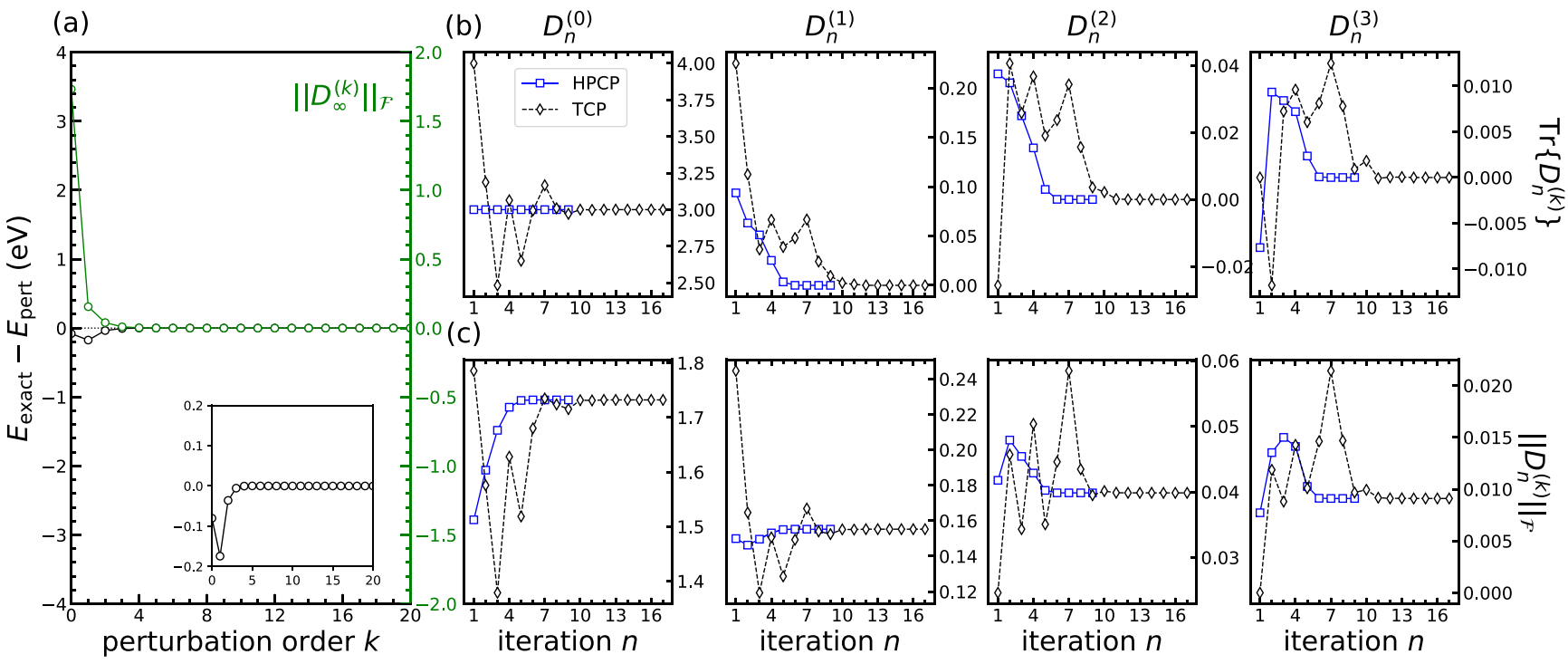

FIG. 2. Perturbation series for $\pi$-bonding in pyridine (see text for details). (a) Convergence of the perturbed energy and the Frobenius norm of the corresponding perturbed density matrices using purification-DMPT. The convergence of the unperturbed and the first three perturbed density matrices during the purification cycles are presented in terms of their traces (b) and their norms (c), for both the TC2 and HPCP polynomials. 
polynomials for the case of benzene should not be overinterpreted since they are merely related to the symmetry of the system. As a matter of fact, for the more general case of pyridine, such property of zero-trace conservation is not observed. We notice that the convergence behavior of the HPCP polynomials is more smooth than TC2 with, after the first few steps a quasi-systematic monotonic approach of the converged perturbed density matrix.

In order to compare the computational performances of the purification, McWeeny and Sylvester DMPT, we have performed a set of calculations on systems of increasing sizes. As for the pyridine case, unperturbed Hückel matrices for aromatic hydrocarbons with increasing ring size were generated along with first-order perturbed Hamiltonians describing the substitution of one of the carbons by a nitrogen atom. In Fig. 3, the central processing unit (CPU) time spent to obtain the unperturbed and first-order perturbed density matrices with respect to the size of the Hückel matrix is plotted. The same tolerance parameter of $\left\|D_{n+1}-D_{n}\right\|_{\mathcal{F}}<10^{-12}$ was used for purifications. We emphasize here that the McWeeny and SylvesterDMPT are direct methods such that provided the eigenstates (or only the unperturbed density matrix for Sylvester-DMPT), the number of FLOPS to solve the DMPT equations is fixed by the size of the problem $M$, whereas for purification-based methods the solutions are found iteratively. In this case, the number of iterations depends on the band-gap of the system, ${ }^{34,80}$ and for HPCP, to a lesser extent, on the filling factor $\theta .^{39}$ In our example, ideal conditions are fulfilled to minimize the number of purifications with $\theta=1 / 2$ and a large HOMO-LUMO gap when compared to the range of the full eigenspectrum. Note that the size of the $\mathrm{N}$-substituted aromatic hydrocarbons does not impact the HOMO-LUMO gap, for which we found, as for pyridine, that the density matrices are converged after 9 and 17 purifications for HPCP and TC2, respectively, independently of $M$. From Fig. 3, we note that the exact diagonalization is more efficient than the purification methods to obtain the unperturbed density matrix. However, once we consider the perturbed state, we observe a net benefit to solve the Sylvester-DMPT equations

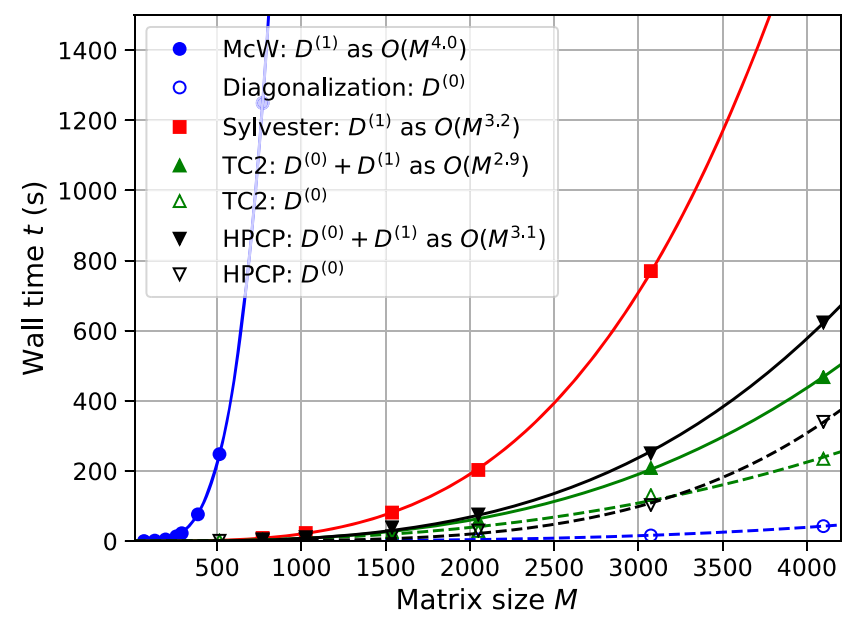

FIG. 3. Scaling performance for the various methods to obtain $D^{(0)}$ and $D^{(1)}$ using a benchmark of Hückel matrices of increasing size. Nonlinear fits of equation $t$ $=\alpha M^{\beta}$ are plotted with solid lines. using the Bartels-Stewart algorithm compared to the sum-overstates approach of McWeeny, which is expected to scale as $O\left(M^{4}\right)$ and $O\left(M^{3}\right)$, respectively. Note that the effective scaling reported in Fig. 3 is in good agreement with these expectations. If we now consider purification methods, which also scales as $O\left(M^{3}\right)$, the computational performances are further improved. We stress that for very low band gaps, these performances are expected to degrade with a CPU time multiplied by a factor 5 at worst. ${ }^{39}$ Finally, it should be mentioned that for purification-DMPT, the plots of Fig. 3 incorporate the calculation time for both $D^{(0)}$ and $D^{(1)}$ (since they cannot be decoupled), whereas for direct methods the former has not been included, increasing the interest for the TC2/HPCP DMPT. Comparison of the TC2 and HPCP shows that despite the more rapid convergence of HPCP, TC2 presents better performances due to its lower number of MMs, which is always (independently of the perturbation order) more than twice less than the one of HPCP. Typically, in our example, TC2 requires two MMs per iteration compared to five for HPCP. However, HPCP requires twice less iterations.

\section{CONCLUSIONS AND PERSPECTIVES}

In this work, we have reviewed three types of density matrix perturbation theory with, for two of them, a resolution of the perturbed density matrices without the support of the unperturbed eigenstates. These two methods, namely, the Sylvester- and purification-DMPT, clearly demonstrate better computational performances compared to the standard sum-over-states approach. This indicates that the current response equation solver as implemented in quantum chemistry codes can be significantly accelerated using those two methods. We have also successfully extended the recursive DMPT proposed by Niklasson and Challacombe to the HPCP polynomial. Compared to TC2, the HPCP-DMPT shows a better stability when approaching convergence, but remains more expensive in terms of computational performance. We emphasize that, under favorable conditions, i.e., for insulators with a filling factor around $1 / 2$, the TCP/HPCP-DMPT clearly outperforms the other approaches. In the near future, we plan to implement the Sylvester- and purification-DMPT using non-orthogonal and perturbation dependent basis set. ${ }^{32,33}$ Within the framework of linear scaling density functional theory as implemented in the CONQUEST code, ${ }^{81}$ this will allow for application to electric and magnetic response calculations at a linear scaling computational cost.

\section{APPENDIX A: SOS-McWEENY DMPT: SECOND AND THIRD ORDERS}

The second-order equation can be derived by applying the resolution of identity to both side of Eq. (13c). Conserving notations of Eq. (17), we obtain

$$
\begin{aligned}
2 D_{o o}^{(2)}+ & D_{o v}^{(2)}+D_{v o}^{(2)}+\left(D^{(1)} D^{(1)}\right)_{o o}+\left(D^{(1)} D^{(1)}\right)_{v v} \cdots \\
& +\left(D^{(1)} D^{(1)}\right)_{o v}+\left(D^{(1)} D^{(1)}\right)_{v o} \\
= & D_{o o}^{(2)}+D_{o v}^{(2)}+D_{v o}^{(2)}+D_{v v}^{(2)}
\end{aligned}
$$

By resolving the product of first-order perturbed density matrices according to 


$$
\begin{aligned}
D^{(1)} D^{(1)} & =D^{(1)} I D^{(1)} \\
& =D^{(1)}(D+\bar{D}) D^{(1)} \\
& =D^{(1)}\left(D^{2}+\bar{D}^{2}\right) D^{(1)},
\end{aligned}
$$

we obtain

$$
\begin{aligned}
& \left(D^{(1)} D^{(1)}\right)_{o o}=D_{o o}^{(1)} D_{o o}^{(1)}+D_{o v}^{(1)} D_{v o}^{(1)}, \\
& \left(D^{(1)} D^{(1)}\right)_{v v}=D_{v v}^{(1)} D_{v v}^{(1)}+D_{v o}^{(1)} D_{o v}^{(1)}, \\
& \left(D^{(1)} D^{(1)}\right)_{o v}=D_{o v}^{(1)} D_{v v}^{(1)}+D_{o o}^{(1)} D_{o v}^{(1)}, \\
& \left(D^{(1)} D^{(1)}\right)_{v o}=D_{v o}^{(1)} D_{o o}^{(1)}+D_{v v}^{(1)} D_{v o}^{(1)} .
\end{aligned}
$$

On inserting the rhs of Eq. (A2) into (A1), and using the properties of Eq. (19), we have

$$
\begin{aligned}
2 D_{o o}^{(2)} & +D_{o v}^{(2)}+D_{v o}^{(2)}+D_{o v}^{(1)} D_{v o}^{(1)}+D_{v o}^{(1)} D_{o v}^{(1)} \\
& =D_{o o}^{(2)}+D_{o v}^{(2)}+D_{v o}^{(2)}+D_{v v}^{(2)} .
\end{aligned}
$$

Therefore, we find

$$
\begin{aligned}
& D_{o o}^{(2)}=-D_{o v}^{(1)} D_{v o}^{(1)}, \\
& D_{v v}^{(2)}=+D_{v o}^{(1)} D_{o v}^{(1)} .
\end{aligned}
$$

Unlike the first-order perturbation, the diagonal components of the second-order perturbed density matrix are likely to be non-zero and can be computed from the first-order perturbed density matrix. Relying furthermore on the symmetry of the perturbed density, it leaves only the occupied-virtual coupling block matrix to evaluate. On resolving the second-order perturbed Hamiltonian matrix using Eq. (14c), we obtain

$$
H_{o v}^{(2)}=\left[H, D_{o v}^{(2)}\right]+\left[H^{(1)}, D^{(1)}\right]_{o v} .
$$

Using the spectral resolution of the non-perturbed Hamiltonian matrix and the perturbed density matrix, Eq. (A5) transforms as

$$
H_{o v}^{(2)}=\sum_{i, j}\left(D_{o v, i j}^{(2)}\left(\epsilon_{i}-\bar{\epsilon}_{j}\right)+\left[H^{(1)}, D^{(1)}\right]_{o v, i j}\right),
$$

which leads to

$$
D_{o v}^{(2)}=\sum_{i, j}\left(\epsilon_{i}-\bar{\epsilon}_{j}\right)^{-1}\left(H_{o v}^{(2)}-\left[H^{(1)}, D^{(1)}\right]_{o v}\right)_{i j} .
$$

The final second-order perturbed density matrix is obtained by summing over $D_{o v}^{(2)}$, its conjugate-transposed and the block-diagonal contributions of Eq. (A4).

Using the same route, the third-order response equation can be derived from Eq. (13d). This yields

$$
\begin{aligned}
2 D_{o o}^{(3)}+ & D_{o v}^{(3)}+D_{v o}^{(3)}+\left(D^{(1)} D^{(2)}\right)_{o o}+\left(D^{(1)} D^{(2)}\right)_{v v} \cdots \\
& +\left(D^{(1)} D^{(2)}\right)_{o v}+\left(D^{(1)} D^{(2)}\right)_{v o} \cdots \\
& +\left(D^{(2)} D^{(1)}\right)_{o o}+\left(D^{(2)} D^{(1)}\right)_{v v} \cdots \\
& +\left(D^{(2)} D^{(1)}\right)_{o v}+\left(D^{(2)} D^{(1)}\right)_{v o} \\
= & D_{o o}^{(3)}+D_{o v}^{(3)}+D_{v o}^{(3)}+D_{v v}^{(3)}
\end{aligned}
$$

where

$$
\begin{aligned}
& \left(D^{(1)} D^{(2)}\right)_{o o}=D_{o o}^{(1)} D_{o o}^{(2)}+D_{o v}^{(1)} D_{v o}^{(2)}, \\
& \left(D^{(1)} D^{(2)}\right)_{v v}=D_{v v}^{(1)} D_{v v}^{(2)}+D_{v o}^{(1)} D_{o v}^{(2)}, \\
& \left(D^{(1)} D^{(2)}\right)_{o v}=D_{o v}^{(1)} D_{v v}^{(2)}+D_{o o}^{(1)} D_{o v}^{(2)}, \\
& \left(D^{(1)} D^{(2)}\right)_{v o}=D_{v o}^{(1)} D_{o o}^{(2)}+D_{v v}^{(1)} D_{v o}^{(2)}, \\
& \left(D^{(2)} D^{(1)}\right)_{o o}=D_{o o}^{(2)} D_{o o}^{(1)}+D_{o v}^{(2)} D_{v o}^{(1)}, \\
& \left(D^{(2)} D^{(1)}\right)_{v v}=D_{v v}^{(2)} D_{v v}^{(1)}+D_{v o}^{(2)} D_{o v}^{(1)}, \\
& \left(D^{(2)} D^{(1)}\right)_{o v}=D_{o v}^{(2)} D_{v v}^{(1)}+D_{o o}^{(2)} D_{o v}^{(1)}, \\
& \left(D^{(2)} D^{(1)}\right)_{v o}=D_{v o}^{(2)} D_{o o}^{(1)}+D_{v v}^{(2)} D_{v o}^{(1)} .
\end{aligned}
$$

From Eqs. (19), (A4) and (A9), Eq. (A8) simplifies to

$$
\begin{aligned}
2 D_{o o}^{(3)}+ & D_{o v}^{(3)}+D_{v o}^{(3)}+D_{o v}^{(1)} D_{v o}^{(2)}+D_{o v}^{(2)} D_{v o}^{(1)} \ldots \\
& +D_{v o}^{(1)} D_{o v}^{(2)}+D_{v o}^{(2)} D_{o v}^{(1)} \\
= & D_{o o}^{(3)}+D_{o v}^{(3)}+D_{v o}^{(3)}+D_{v v}^{(3)} .
\end{aligned}
$$

On identifying the lhs and rhs terms, it follows that

$$
\begin{aligned}
& D_{o o}^{(3)}=-\left(D_{o v}^{(1)} D_{v o}^{(2)}+D_{o v}^{(2)} D_{v o}^{(1)}\right), \\
& D_{v v}^{(3)}=+\left(D_{v o}^{(1)} D_{o v}^{(2)}+D_{v o}^{(2)} D_{o v}^{(1)}\right) .
\end{aligned}
$$

Again, the last equation shows that the diagonal components are computed with the first and second-order perturbed density matrices. At this point, we emphasize that only the occupied-virtual transition matrix needs to be evaluated since the perturbed density matrix is Hermitian.

Relying on the spectral resolution, the third-order perturbed Hamiltonian matrix is given by

$$
H_{o v}^{(3)}=\left[H, D_{o v}^{(3)}\right]+\left[H^{(1)}, D^{(2)}\right]_{o v}+\left[H^{(2)} D^{(1)}\right]_{o v},
$$

$$
\begin{aligned}
= & \sum_{i, j} D_{o v, i j}^{(3)}\left(\epsilon_{i}-\bar{\epsilon}_{j}\right) \\
& \times\left(\left[H^{(1)}, D^{(2)}\right]_{o v}+\left[H^{(2)} D^{(1)}\right]_{o v}\right)_{i j},
\end{aligned}
$$

which leads to

$$
\begin{aligned}
D_{o v}^{(3)}= & \sum_{i, j}\left(\epsilon_{i}-\bar{\epsilon}_{j}\right)^{-1} \\
& \times\left(H_{o v}^{(3)}-\left[H^{(1)}, D^{(2)}\right]_{o v}-\left[H^{(2)}, D^{(1)}\right]_{o v}\right)_{i j} .
\end{aligned}
$$

By summing over contributions of Eqs. (A11) and (A13) and its conjugate-transposed, we obtain the third-order perturbed density matrix. It is worth mentioning that the direct resolutions of the second or third-order perturbed quantities implies prior knowledge of the lower orders (first and second, respectively), in such a way that, whatever is the order to be resolved, i.e., Eqs. (A11) and (A13) on 
one side or Eqs. (A4) - (A7) on the other side, both cases are involved to solve for the linear-response of the order $(k-1)$ to obtain the perturbed quantities at the order $k$. Eventually, by mathematical induction, it is straightforward to generalize these working equations to any order $k$, as introduced in Eqs. (27)-(31).

\section{APPENDIX B: ALTERNATIVE FORMULATION OF DMPT}

In this appendix, we shall show that the plane wave-based DMPT introduced by Lazzeri and Mauri ${ }^{69}$ is closely related to the atomic-orbital based DMPT method of Kussmann and Ochsenfeld $^{26,27}$ (and by extension the Sylvester-DMPT), both relying on the CG minimization. Hereafter, they will be referred to as PW-DMPT and AO-DMPT, respectively.

As discussed in Sec. II D, employing a PW basis set with $M_{\mathrm{PW}} \gg N$ constrains us to use iterative diagonalization, where, for an insulator, the $N$ first (lowest) eigenstates, $\left\{\epsilon_{i}, \psi_{i}\right\}_{i \in \text { occ }}^{N}$, necessary and sufficient to obtain the unperturbed ground-state, can be determined with a satisfying accuracy. Here, we will assume the linear-DMPT regime: in order to determine the $k$ th-order perturbed density, all the preceding orders, up to $(k-1)$, are known. As a result, for a PW basis set, at the zero order, the unperturbed density matrix, $D^{(0)} \equiv D$, can be resolved in terms of the occupied states following Eq. (4), and knowing only the occupied eigenstates, the unperturbed Hamiltonian matrix, $H^{(0)} \equiv H$, can be formally expressed as

$$
H=\sum_{i \in \mathrm{occ}}\left|\psi_{i}\right\rangle \epsilon_{i}\left\langle\psi_{i}\right|+\bar{D} H \bar{D},
$$

recalling that, from the closure relation of Eq. (9), $\bar{D}=I-D$. Within the framework of the AO-DMPT method, the $k$-order perturbed density matrix, $D^{(k)}$, is found as a solution of the following equation:

$$
\left[D, \sum_{l=0}^{k}\left[H^{(l)}, D^{(k-l)}\right]\right]=0 .
$$

It must be emphasized that, in comparison to the McWeeny-DMPT and Eq. (22), or the PW-DMPT and Eq. (B1), the resolution of the AO-DMPT equation is free of any intermediate spectral resolution of the unperturbed Hamiltonian matrix. Indeed, the PW-DMPT may be viewed as an intermediate strategy between the former and the later, where Eq. (B2) is decomposed to perform an occupiedperturbed state-by-state resolution. ${ }^{69}$ For instance, by applying to Eq. (B2) the identity $[D, O]=D O \bar{D}-\bar{D} O D$, and projecting to the right on $\left|\psi_{i}\right\rangle$ with $i \in$ occ, we find

$$
\bar{D} \sum_{l=0}^{k}\left[H^{(l)}, D^{(k-l)}\right]\left|\psi_{i}\right\rangle=0 .
$$

By extracting the terms containing the $k$ th-order density matrix from the sum, and by reordering, it appears

$$
\left(\bar{D} H D^{(k)}-\bar{D} D^{(k)} H\right)\left|\psi_{i}\right\rangle=-\sum_{l=1}^{k} \bar{D}\left[H^{(l)}, D^{(k-l)}\right]\left|\psi_{i}\right\rangle .
$$

On substitution of the expression of $H$ from Eq. (B1) into Eq. (B4), and recalling that, at the zero temperature limit, the converged ground-state one-electron and one-hole density matrices must respect the idempotency and the stationary conditions of Eqs. (13a) and (14a), respectively, we arrive at

$$
\left(H \bar{D} D^{(k)}-\bar{D} D^{(k)} \epsilon_{i}\right)\left|\psi_{i}\right\rangle=-\sum_{l=1}^{k} \bar{D}\left[H^{(l)}, D^{(k-l)}\right]\left|\psi_{i}\right\rangle .
$$

Complying with the notations of Ref. 69 by introducing

$$
\begin{gathered}
\left|\eta_{i}^{(k)}\right\rangle=\bar{D} D^{(k)}\left|\psi_{i}\right\rangle, \\
\text { such that } D_{v o}^{(k)}=\sum_{i \in \mathrm{occ}}\left|\eta_{i}^{(k)}\right\rangle\left\langle\psi_{i}\right|,
\end{gathered}
$$

we recover Eq. (13) of the article, that is,

$$
\left(H-I \epsilon_{i}\right)\left|\eta_{i}^{(k)}\right\rangle=-\sum_{l=1}^{k} \bar{D}\left[H^{(l)}, D^{(k-l)}\right]\left|\psi_{i}\right\rangle .
$$

In the interests of completeness, the SOS Eq. (29) can be found back by further resolving the full spectrum of $H$. By using Eq. (22), along with the resolution of identity (9), the rhs of Eq. (B7) transforms as

$$
\begin{aligned}
\left(H-\epsilon_{i} I\right)\left|\eta_{i}^{(k)}\right\rangle= & \sum_{i^{\prime} \in \mathrm{occ}}\left|\psi_{i^{\prime}}\right\rangle\left(\epsilon_{i^{\prime}}-\epsilon_{i}\right)\left\langle\psi_{i^{\prime}} \mid \eta_{i}^{(k)}\right\rangle \\
& +\sum_{j^{\prime} \in \text { virt }}\left|\bar{\psi}_{j^{\prime}}\right\rangle\left(\bar{\epsilon}_{j^{\prime}}-\epsilon_{i}\right)\left\langle\bar{\psi}_{j^{\prime}} \mid \eta_{i}^{(k)}\right\rangle .
\end{aligned}
$$

By remarking that (i) $\left\langle\psi_{i^{\prime}} \mid \eta_{i}^{(k)}\right\rangle=0 \quad \forall i^{\prime}$ and (ii) $\left\langle\bar{\psi}_{j^{\prime}} \mid \eta_{i}^{(k)}\right\rangle$ $=\left\langle\bar{\psi}_{j^{\prime}}\left|D^{(k)}\right| \psi_{i}\right\rangle$, we found that Eq. (B8) simplifies to

$$
\left(H-\epsilon_{i} I\right)\left|\eta_{i}^{(k)}\right\rangle=\sum_{j \in \text { virt }}\left|\bar{\psi}_{j}\right\rangle\left(\bar{\epsilon}_{j}-\epsilon_{i}\right)\left\langle\bar{\psi}_{j}\left|D^{(k)}\right| \psi_{i}\right\rangle .
$$

Inserting the above on the lhs of Eq. (B7) and resolving the one-hole density matrix of the rhs, we have

$$
\begin{aligned}
& \sum_{j \in \text { virt }}\left(\bar{\epsilon}_{j}-\epsilon_{i}\right)\left|\bar{\psi}_{j}\right\rangle\left\langle\bar{\psi}_{j}\left|D^{(k)}\right| \psi_{i}\right\rangle \\
& =\sum_{l=1}^{k} \sum_{j \in \mathrm{virt}}\left|\bar{\psi}_{j}\right\rangle\left\langle\bar{\psi}_{j}\left|\left[D^{(k-l)}, H^{(l)}\right]\right| \psi_{i}\right\rangle,
\end{aligned}
$$

which further gives the analytical expression of $\left|\eta_{i}^{(k)}\right\rangle$ with respect to the lower order perturbed density matrices,

$$
\left|\eta_{i}^{(k)}\right\rangle=\sum_{l=1}^{k} \sum_{j \in \mathrm{virt}} \frac{\left\langle\bar{\psi}_{j}\left|\left[D^{(k-l)}, H^{(l)}\right]\right| \psi_{i}\right\rangle}{\bar{\epsilon}_{j}-\epsilon_{i}}\left|\bar{\psi}_{j}\right\rangle .
$$

Following definitions (B6), by summing over the $N$ perturbed projectors, this yields the $k$ th virtual-occupied transition matrix

$$
D_{v o}^{(k)}=\sum_{l=1}^{k} \sum_{i \in \text { occ }} \sum_{j \in \operatorname{virt}} \frac{\left\langle\bar{\psi}_{j}\left|\left[D^{(k-l)}, H^{(l)}\right]\right| \psi_{i}\right\rangle}{\bar{\epsilon}_{j}-\epsilon_{i}}\left|\bar{\psi}_{j}\right\rangle\left\langle\psi_{i}\right|,
$$

which is the conjugate transpose of the McWeeny Eq. (29). Note that, for $k>1$, the remaining occupied-occupied and virtualvirtual components necessary to build the full $k$ th-order matrix, i.e., 
$D^{(k)}=D_{o v}^{(k)}+D_{v o}^{(k)}+D_{o o}^{(k)}+D_{v v}^{(k)}$, can be easily computed from the lowest orders using Eqs. (30) and (31).

\section{DATA AVAILABILITY}

The data that support the findings of this study are available from the corresponding author upon reasonable request.

\section{REFERENCES}

${ }^{1}$ A. Weiße, G. Wellein, A. Alvermann, and H. Fehske, Rev. Mod. Phys. 78, 275 (2006).

${ }^{2}$ R. M. Stevens, R. M. Pitzer, and W. N. Lipscomb, J. Chem. Phys. 38, 550 (1963).

${ }^{3}$ J. Gerratt and I. M. Mills, J. Chem. Phys. 49, 1719 (1968).

${ }^{4}$ J. Gerratt and I. M. Mills, J. Chem. Phys. 49, 1730 (1968).

${ }^{5} \mathrm{~K}$. Thomsen and P. Swanstrøm, Mol. Phys. 26, 735 (1973).

${ }^{6}$ R. Ditchfield, Mol. Phys. 27, 789 (1974).

${ }^{7}$ J. A. Pople, R. Krishnan, H. B. Schlegel, and J. S. Binkley, Int. J. Quantum Chem. 16, 225 (1979).

${ }^{8}$ M. Frisch, M. Head-Gordon, and J. Pople, Chem. Phys. 141, 189 (1990).

${ }^{9}$ Y. Osamura, Y. Yamaguchi, P. Saxe, D. J. Fox, M. A. Vincent, and H. F. Schaefer, J. Mol. Struct.: THEOCHEM 103, 183 (1983).

${ }^{10}$ R. McWeeny, Phys. Rev. 126, 1028 (1962).

${ }^{11}$ R. McWeeny, Chem. Phys. Lett. 1, 567 (1968).

${ }^{12}$ G. Diercksen and R. McWeeny, J. Chem. Phys. 44, 3554 (1966).

${ }^{13}$ J. A. Pople, Trans. Faraday Soc. 49, 1375 (1953).

${ }^{14}$ R. Pariser and R. G. Parr, J. Chem. Phys. 21, 767 (1953).

${ }^{15}$ R. Pariser and R. G. Parr, J. Chem. Phys. 21, 466 (1953).

${ }^{16}$ R. Moccia, Theor. Chim. Acta 8, 192 (1967).

${ }^{17}$ R. Moccia, Chem. Phys. Lett. 5, 260 (1970).

${ }^{18}$ J. L. Dodds, R. McWeeny, and A. J. Sadlej, Mol. Phys. 34, 1779 (1977).

${ }^{19}$ J. L. Dodds, R. McWeeny, W. T. Raynes, and J. P. Riley, Mol. Phys. 33, 611 (1977).

${ }^{20}$ K. Woliński and A. J. Sadlej, Mol. Phys. 41, 1419 (1980).

${ }^{21}$ K. Wolinski, J. F. Hinton, and P. Pulay, J. Am. Chem. Soc. 112, 8251 (1990).

${ }^{22}$ C. Ochsenfeld and M. Head-Gordon, Chem. Phys. Lett. 270, 399 (1997).

${ }^{23}$ X.-P. Li, R. W. Nunes, and D. Vanderbilt, Phys. Rev. B 47, 10891 (1993).

${ }^{24}$ R. McWeeny, Proc. R. Soc. London, Ser. A 235, 496 (1956).

${ }^{25}$ R. McWeeny, Rev. Mod. Phys. 32, 335 (1960).

${ }^{26}$ J. Kussmann and C. Ochsenfeld, J. Chem. Phys. 127, 054103 (2007).

${ }^{27}$ J. Kussmann and C. Ochsenfeld, J. Chem. Phys. 127, 204103 (2007).

${ }^{28}$ H. Larsen, T. Helgaker, J. Olsen, and P. Jørgensen, J. Chem. Phys. 115, 10344 (2001).

${ }^{29}$ S. Coriani, S. Høst, B. Jansík, L. Thøgersen, J. Olsen, P. Jørgensen, S. Reine, F. Pawłowski, T. Helgaker, and P. Sałek, J. Chem. Phys. 126, 154108 (2007).

${ }^{30}$ T. Helgaker, H. Larsen, J. Olsen, and P. Jørgensen, Chem. Phys. Lett. 327, 397 (2000).

${ }^{31}$ A. M. N. Niklasson and M. Challacombe, Phys. Rev. Lett. 92, 193001 (2004).

${ }^{32}$ A. M. N. Niklasson, V. Weber, and M. Challacombe, J. Chem. Phys. 123, 044107 (2005).

${ }^{33}$ A. M. N. Niklasson and V. Weber, J. Chem. Phys. 127, 064105 (2007).

${ }^{34}$ A. M. N. Niklasson, Phys. Rev. B 66, 155115 (2002)

${ }^{35}$ M. J. Cawkwell, E. J. Sanville, S. M. Mniszewski, and A. M. N. Niklasson, J. Chem. Theory Comput. 8, 4094 (2012).

${ }^{36}$ M. J. Cawkwell, M. A. Wood, A. M. N. Niklasson, and S. M. Mniszewski, J. Chem. Theory Comput. 10, 5391 (2014).

${ }^{37}$ V. Weber, A. M. N. Niklasson, and M. Challacombe, Phys. Rev. Lett. 92, 193002 (2004).

${ }^{38}$ V. Weber, A. M. N. Niklasson, and M. Challacombe, J. Chem. Phys. 123, 044106 (2005).

${ }^{39}$ L. A. Truflandier, R. M. Dianzinga, and D. R. Bowler, J. Chem. Phys. 144, 091102 (2016).
${ }^{40}$ S. Goedecker and M. Teter, Phys. Rev. B 51, 9455 (1995).

${ }^{41}$ R. Baer and M. Head-Gordon, Phys. Rev. Lett. 79, 3962 (1997).

${ }^{42}$ J. O. Hirschfelder, W. B. Brown, and S. T. Epstein, Advances in Quantum Chemistry (Elsevier, 1964), Vol. 1, pp. 255-374.

${ }^{43} \mathrm{~T}$. Helgaker, P. Jørgensen, and J. Olsen, Molecular Electronic-Structure Theory (John Wiley \& Sons, Ltd., London, 2000).

${ }^{44}$ P. Pulay, J. Chem. Phys. 78, 5043 (1983).

${ }^{45}$ H. Sekino and R. J. Bartlett, J. Chem. Phys. 85, 976 (1986).

${ }^{46}$ S. P. Karna and M. Dupuis, J. Comput. Chem. 12, 487 (1991).

${ }^{47}$ F. Furche, J. Chem. Phys. 114, 5982 (2001).

${ }^{48}$ M. Kobayashi, T. Touma, and H. Nakai, J. Chem. Phys. 136, 084108 (2012).

${ }^{49} \mathrm{~J}$. Kußmann, Development of Linear Scaling ab initio Methods based on Electron Density Matrices (Universität Tübingen, Germany, 2006).

${ }^{50}$ The passing from $\left[D,\left[H, D^{(1)}\right]\right]+\left[D,\left[H^{(1)}, D\right]=0\right.$ to Eq. (32) required the use of the identity: $[A,[B, C]]=[B,[A, C]]+[C,[B, A]]$.

${ }^{51} \mathrm{~J}$. Brandts, in Large-Scale Scientific Computing, edited by S. Margenov, J. Waśniewski, and P. Yalamov (Springer Berlin Heidelberg, Berlin, Heidelberg, 2001), pp. 462-470.

${ }^{52}$ V. Simoncini, SIAM Rev. 58, 377 (2016).

${ }^{53}$ Note that the special case where $B=A^{t}$ in the Sylvester equation $A X+X B=C$ is also referred to as the Lyapunov equation in litterature. ${ }^{5}$

${ }^{54}$ R. H. Bartels and G. W. Stewart, Commun. ACM 15, 820 (1972).

${ }^{55} \mathrm{Y}$. Saad, Iterative Methods for Sparse Linear Systems, Other Titles in Applied Mathematics (Society for Industrial and Applied Mathematics, 2003).

${ }^{56}$ J. Nocedal and S. Wright, Numerical Optimization, Springer Series in Operations Research and Financial Engineering, 2nd ed. (Springer-Verlag, New York, 2006).

${ }^{57}$ E. R. Davidson, J. Comput. Phys. 17, 87 (1975).

${ }^{58}$ D. M. Wood and A. Zunger, J. Phys. A: Math. Gen. 18, 1343 (1985).

${ }^{59} \mathrm{G}$. Kresse and J. Furthmüller, Phys. Rev. B 54, 11169 (1996).

${ }^{60}$ M. P. Teter, M. C. Payne, and D. C. Allan, Phys. Rev. B 40, 12255 (1989).

${ }^{61}$ M. C. Payne, M. P. Teter, D. C. Allan, T. A. Arias, and J. D. Joannopoulos, Rev. Mod. Phys. 64, 1045 (1992).

${ }^{62}$ P. Giannozzi, S. Baroni, N. Bonini, M. Calandra, R. Car, C. Cavazzoni, D. Ceresoli, G. L. Chiarotti, M. Cococcioni, I. Dabo, A. Dal Corso, S. de Gironcoli, S. Fabris, G. Fratesi, R. Gebauer, U. Gerstmann, C. Gougoussis, A. Kokalj, M. Lazzeri, L. Martin-Samos, N. Marzari, F. Mauri, R. Mazzarello, S. Paolini, A. Pasquarello, L. Paulatto, C. Sbraccia, S. Scandolo, G. Sclauzero, A. P. Seitsonen, A. Smogunov, P. Umari, and R. M. Wentzcovitch, J. Phys.: Condens. Matter 21, 395502 (2009).

${ }^{63} \mathrm{G}$. Kresse and J. Furthmüller, Comput. Mater. Sci. 6, 15 (1996).

${ }^{64}$ X. Gonze, Phys. Rev. A 52, 1096 (1995).

${ }^{65}$ X. Gonze, Phys. Rev. B 55, 10337 (1997)

${ }^{66}$ S. Baroni, P. Giannozzi, and A. Testa, Phys. Rev. Lett. 58, 1861 (1987).

${ }^{67}$ S. Baroni, S. de Gironcoli, A. Dal Corso, and P. Giannozzi, Rev. Mod. Phys. 73, 515 (2001).

${ }^{68}$ M. Lazzeri and F. Mauri, Phys. Rev. Lett. 90, 036401 (2003).

${ }^{69}$ M. Lazzeri and F. Mauri, Phys. Rev. B 68, 161101 (2003).

${ }^{70}$ A. M. N. Niklasson, in Linear-Scaling Techniques in Computational Chemistry and Physics, Challenges and Advances in Computational Chemistry and Physics No. 13, edited by R. Zalesny, M. G. Papadopoulos, P. G. Mezey, and J. Leszczynski (Springer Netherlands, 2011), pp. 439-473.

${ }^{71}$ S. Geršgorin, Proc. USSR Acad. Sci. 51, 749 (1931); available at http://www. mathnet.ru/php/archive.phtml?wshow=paper\&jrnid=im\&paperid=5235\&option lang=eng.

${ }^{72}$ Even if obvious, we recall that: $\operatorname{sign}(x)=2 \Theta(x)-1$. The TC2 formulation of Eq. (40a) using $\Theta(x)$ instead of the sign $(x)$ is only useful when compared with the HPCP polynomial (43).

${ }^{73}$ E. H. Rubensson, SIAM J. Sci. Comput. 34, B1 (2012).

${ }^{74}$ E. H. Rubensson, E. Rudberg, and P. Sałek, J. Chem. Phys. 128, 074106 (2008). 
${ }^{75}$ A. H. R. Palser and D. E. Manolopoulos, Phys. Rev. B 58, 12704 (1998).

${ }^{76}$ D. A. Mazziotti, Phys. Rev. E 68, 066701 (2003).

${ }^{77}$ Maybe it is worth to recall that for square matrices of size $M \times M$, the number of floating-point operations (FLOPs) for dense matrix-matrix multiplication scales as $O\left(M^{3}\right)$, compare to $O\left(M^{2}\right)$ for all the other matrix operations involved in those perturbed recursions, e.g. scalar multiplication, addition and transposition; available at http://www.mathnet.ru/php/archive.phtml?wshow=paper\&jrnid=im\& paperid=5235\&option_lang=eng.
${ }^{78}$ For $k$ odd, exactly $(k+1) / 2$ MMs must be performed for the TC2 vs $(3 k+3) / 2$ for the HPCP. For $k$ even, it is found $(k+2) / 2$ vs $(3 k+4) / 2 \mathrm{MMs}$, respectively.

${ }^{79}$ T. A. Albright, J. K. Burdett, and M. Whangbo, Orbital Interactions in Chemistry (John Wiley \& Sons, Ltd., 1985).

${ }^{80}$ E. Rudberg and E. H. Rubensson, J. Phys.: Condens. Matter 23, 075502 (2011).

${ }^{81}$ A. Nakata, J. S. Baker, S. Y. Mujahed, J. T. L. Poulton, S. Arapan, J. Lin, Z. Raza, S. Yadav, L. Truflandier, T. Miyazaki, and D. R. Bowler, J. Chem. Phys. 152, $164112(2020)$. 